\title{
COMPOSIÇÃO DE ESPÉCIES DA ICTIOFAUNA DO CÓRREGO SEVERO ALTA FLORESTA-MT
}

\author{
Reginaldo Carvalho dos Santos ${ }^{1}$, Andreia Aparecida Franco ${ }^{2}$, Vanuza Aparecida \\ Martins de Oliveira ${ }^{3}$, Maicon Diego Pinto Rossi ${ }^{4}$, Cristiano de O. Schuingues ${ }^{5}$
}

1Biólogo - Mestrando do Programa de Biodiversidade e Agrossistemas AmazônicosLaboratório de Zoologia e Morfologia da Universidade do Estado de Mato Grosso -

Alta Floresta. email: carvalhotnn@gmail.com

2Bióloga - Mestranda do Programa de Biodiversidade e Agrossistemas Amazônicos

- Universidade do Estado de Mato Grosso - Alta Floresta.

3Bióloga - Esp. LIAM - Laboratório Ictiologia da Amazônia Meridional

4Biólogo - Mestrando do Programa de Biodiversidade e Agrossistemas Amazônico Laboratório de Zoologia da Universidade do Estado de Mato Grosso - Alta Floresta. 5Biólogo - Mestre em de Biodiversidade e Agrossistemas Amazônicos - Laboratório de Zoologia e Morfologia da Universidade do Estado de Mato Grosso-Alta Floresta.

\section{Recebido em: 02/10/2017 - Aprovado em: 21/11/2017 - Publicado em: 05/12/2017 DOI: 10.18677/EnciBio_2017B77}

\section{RESUMO}

Objetivou neste trabalho realizar a composição da ictiofauna do córrego do SeveroAF. No Brasil são registradas 2.587 espécies de peixes e esta grande diversidade da ictiofauna é atribuída a grande quantidade de ambientes aquáticos encontrados na Amazônia, que vem sofrendo com a antropização. O córrego do Severo tem sua nascente na área rural do município de Alta Floresta, com largura média de quatro metros percorrendo o perímetro urbano por treze quilômetros. As coletas foram realizadas em setembro de 2012, em quatro pontos no córrego Severo. Para as coletas foram utilizados puçás, rede de arrasto e redes de espera durante o período de 60 minutos, os exemplares capturados foram acondicionados em formol $10 \%$, após a fixação os peixes foram transferidos para serem conservados em etanol $70 \%$. Foram coletados 539 indivíduos distribuídos em 39 espécies. A ordem mais abundante foi a characiforme. Os peixes de pequeno porte se adaptam melhor a ambientes antropizados, sendo essas espécies são de hábitos natatórios intensos, além de possuírem dieta onívora. Para a manutenção da biodiversidade aquática é necessária a preservação das margens riparias do córrego Severo, desta forma condicionando uma melhora no ecossistema aquático e preservando a biodiversidade.

PALAVRAS-CHAVE: antropização, biodiversidade, peixes chariciformes. 


\title{
TITLE:COMPOSITION OF ICHTHYOFAUNA SPECIES IN SEVERE HIGH FOREST STREAM-MT
}

\begin{abstract}
Objective in this work to perform the composition of the ichthyofauna of the SeveroAF stream. In Brazil 2,587 species of fish are registered. This great diversity of the ichthyofauna is attributed to the great amount of aquatic environments found in the Amazon, which has been suffering from anthropization. The stream of the Severo has its source in the rural area of the municipality of Alta Floresta, with average width of four meters walking the urban perimeter for thirteen kilometers. The collections were carried out in September 2012, at four points in the Severo stream. Samples were collected in $10 \%$ formaldehyde, after fixation, the fish were transferred to be stored in $70 \%$ ethanol. A total of 539 individuals were collected from 39 species. The most abundant order was characiform. Small fishes are better adapted to anthropic environments, these species are of intense swimming habits, besides having an omnivorous diet. For the maintenance of aquatic biodiversity, it is necessary to preserve the riparian banks of the Severo stream, thus conditioning an improvement in the aquatic ecosystem and preserving biodiversity.
\end{abstract}

KEYWORDS: Anthropization, biodiversity, chariciform fish.

\section{INTRODUÇÃO}

A região neotropical possui a maior diversidade ictiológica do mundo (REIS, 2013), sendo que a família Characidae apresenta maior diversidade de espécies da região neotropical (sensu MIRANDE, 2010), essa alta diversidade é atribuída a diversos sistemas hidrográficos (MACHADO, 2008), sendo que esses ambientes fornecem maiores quantidades de recursos e de habitats (TOWNSEND et al., 2010). No Brasil para ictiofauna de água doce são registradas cerca de 2.587 espécies, que estão distribuídas em 39 famílias (BUCKUP et al., 2007).

Os igarapés são ecossistemas aquáticos lóticos de pequeno porte na Amazônia, são numerosos e são considerados ambientes frágeis devido sua dependência da floresta riparia (BOJSEN ; BARRIGA, 2002). Floresta ripária é de suma importância, pois mantém a integridade dos sistemas aquáticos, controlando o fluxo de água e fornecendo abrigo e alimentação para os organismos aquáticos (BARRELA et al., 2001, CASATTI, 2010).

Antropização de áreas ripárias promovem mudanças de habitats e alterações na disponibilidade de recursos alimentares para ictiofauna, caracterizando-se como uma grave ameaça a diversidade aquática (LORION; KENNEDY, 2009; CASATTI, 2010; SILVA et al., 2012), acarretando o aumento de sedimentos, maior luminosidade no curso do rio, promovendo a diminuição de espécies intolerantes e aumento de espécies generalistas (LORION ; KENNEDY, 2009; FERREIRA, 2010).

O desmatamento de matas ripárias influencia na comunidade de peixes, pois são responsáveis pela diminuição de micro habitats para reprodução, abrigo e alimentação dos peixes (CASSATTI et al., 2009). Na tentativa de subsidiar estratégias de conservação e recuperação ambiental é necessário a interação dos conhecimentos taxonômicos da ictiofauna e compreender a sua relação com o ambiente (KARR ; DUDLEY, 1981; FERREIRA ; CASSATTI, 2006). Neste contexto, o objetivo deste trabalho foi analisar a composição de espécies da ictiofauna do córrego Severo localizado no município de Alta Floresta, Mato Grosso. 


\section{MATERIAL E MÉTODOS}

\section{Área de Estudo}

O município de Alta Floresta, com área de 8.976,309 km² (IBGE, 2016), está situado na porção norte do estado de Mato Grosso possuindo uma população de 50.082 , nas coordenadas geográficas $56.08^{\circ}$ de longitude oeste e $9.87^{\circ}$ de longitude sul distante $830 \mathrm{~km}$ da capital Cuiabá.

O córrego Severo tem a nascente na área rural do município de Alta Floresta, é um rio de segunda ordem, percorrendo o perímetro urbano do município de Alta Floresta por aproximadamente treze quilômetros, com largura média de quatro metros. Foram realizadas coletas em quatro trechos ao longo do córrego, sendo o trecho 1 na nascente e 3 trechos de coleta no perímetro urbano no município de Alta Floresta (Figura 1).

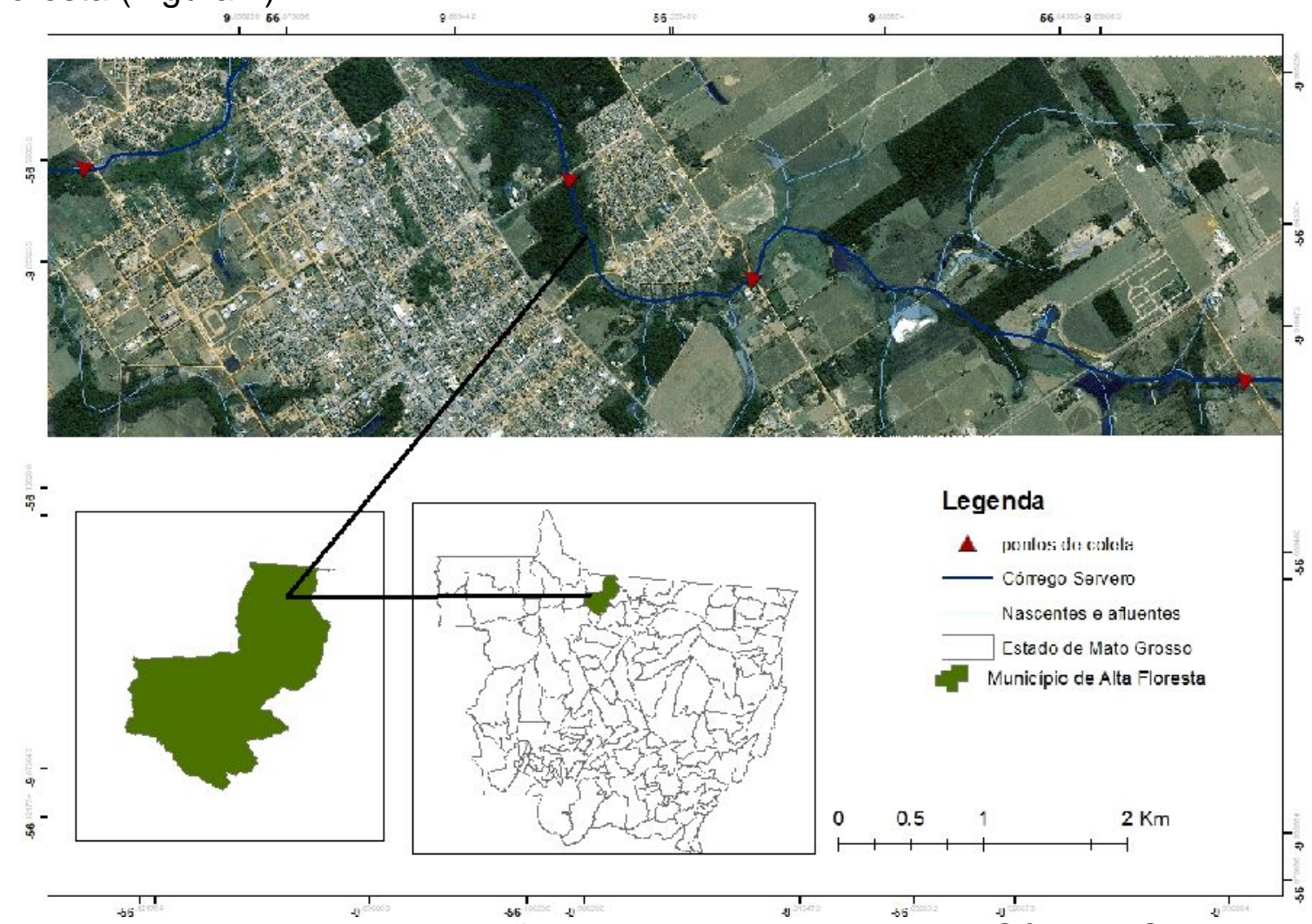

FIGURA 1. Mapa de localizaçã̃o dos pontos de coleta do Córrego Severo no município de Alta Floresta - MT.

\section{Coleta de dados}

As coletas foram realizadas em setembro de 2012. Foram utilizadas redes com $2,5 \mathrm{~cm}$ entre nós para fechar o local de coleta. Para a captura dos peixes foram utilizadas tarrafas com malhas variadas $(1 \mathrm{~cm}, 2,5 \mathrm{~cm})$, redes de espera com diferentes malhadeiras (entre nós opostos de $1 \mathrm{~cm}, 2,5 \mathrm{~cm}$ ), bem como puçás para coletar os exemplares de menor tamanho e rede de arrasto. Foram efetuados 60 minutos de coleta com os puçás, redes de arrasto e espera e tarrafas na área delimitada.

Os exemplares capturados foram acondicionados em recipientes contendo formalina $10 \%$ para a fixação e após 72 horas, os peixes forma transferidos para solução conservante de etanol $70 \%$. Posteriormente os exemplares foram ENCICLOPÉDIA BIOSFERA, Centro Científico Conhecer - Goiânia, v. 14 n.26; p.903 
identificados através das chaves de identificação de (GÉRY, 1977; REIS et al., 2003). Os exemplares coletados foram depositados no Laboratório de Ictiologia da Amazônia Meridional (LIAM) - UNEMAT, Campus de Alta Floresta-MT.

A diversidade de espécies foi obtida através do índice de diversidade de Shannon-Wienner (H'), o agrupamento UPGMA, índices foram calculado pelo pacote estatístico (PAST).

\section{RESULTADOS E DISCUSSÃO}

Foram coletadas 39 espécies totalizando 597 indivíduos, distribuídos nas ordens Characiformes, Siluriformes, Perciformes e Gymnotiformes, sendo os characiformes os mais representativos com 24 espécies, os siluriformes com oito espécies, perciformes com quatro espécies e gymnotiformes com três espécies. 0 trecho 2 apresentou maior riqueza com 31 espécies e maior abundância de indivíduos, sendo a espécie Hemigramus cf. marginatus foi a mais abundante (Tabela 1). A maior diversidade de espécies foi observada no trecho 2, com a maior Equitabilidade no trecho 3 de coleta (Figura 2). $\mathrm{O}$ trecho 2, apresentou maior diversidade observada e diversidade esperada (Figura 3).

TABELA 1. Táxons amostrados no córrego Severo Alta Floresta-MT, em diferentes trechos de coleta e número e espécies coletadas por trecho de coleta.

\begin{tabular}{|c|c|c|c|c|}
\hline \multirow{2}{*}{\multicolumn{5}{|c|}{$\begin{array}{l}\text { Ordem Characiformes } \\
\text { Família Acestrorhynchidae }\end{array}$}} \\
\hline & & & & \\
\hline Acestrorhynchus microlepis & & 4 & & \\
\hline \multicolumn{5}{|l|}{ Família Anostomidae } \\
\hline Leporinus friderici & & 1 & & \\
\hline Leporinus vanzoi & & 5 & & \\
\hline $\begin{array}{l}\text { Leporinus fasciatus } \\
\text { Família Characidae }\end{array}$ & 1 & & & \\
\hline Bryconops transitoria & 1 & 5 & & 8 \\
\hline Thayeria boehklei & & 2 & & \\
\hline Myleus setiger & & 2 & & \\
\hline Moenkausia collettii & & 2 & & 1 \\
\hline Phenacogaster pectinatus & 5 & 2 & & 1 \\
\hline Hyphessobrycon gr. bentosi & & 4 & & \\
\hline Hemigramus cf. marginatus & 15 & 57 & & \\
\hline Hemigramus ocellifer & 1 & & & 1 \\
\hline Astyanax mutidens & 3 & 72 & & 11 \\
\hline Brachychalcinus orbiculares & & & & 3 \\
\hline Hyphessobrycon heliacus & 86 & & & \\
\hline Jupiaba polyleps & & 4 & & 1 \\
\hline $\begin{array}{l}\text { Creagrutus sp. } \\
\text { Familia Poeciliidae }\end{array}$ & & 1 & & \\
\hline Poecilia reticulata & & 2 & 3 & \\
\hline Família Ctenoluciidae & & & & \\
\hline Characidium zebra & & 9 & 1 & \\
\hline
\end{tabular}


Família Curimatidae

Cyphocharax leucosticus

Cyphocharax spiluropsis

Steindachenerina fasciata

Família Erythriniidae

Hoplias malabaricus

2

15

6

4

2

Família Hemiodontidae

Hemiodus microlepis

Ordem Gymnotiformes

Família Hypopomidae

Gymnotus carapo

11

2

Família Sternopygidae

Eigenmannia limbata

Sternopygus macrurus

$8 \quad 13$

Ordem Perciformes

Família Cichlidae

Crenicichla inpa

Crenicichla lepidota

Cichla mirianae

83

Aequidens epae

$\begin{array}{ll}6 & 2 \\ 6 & 1 \\ & 1\end{array}$

1

Ordem Siluriformes

Família Callichthyidae

Corydoras xinguensis

Família Loricariidae

Hypostomus soniae

Hypostomus faveolus

Otocinclus hasemani

Rineloricaria sp.

2

Família Trichomycteridae

Ituglanis amazonicus

1

Família Auchenipteridae

Parauchenipterus galeatus

Familia Heptapteridae

Imparfinis stictonotus

1

8

1

TABELA 2: Índices estatísticos das espécies coletadas no Córrego do Severo.

\begin{tabular}{lcccc}
\hline Índices & Trecho 1 & Trecho 2 & Trecho 3 & Trecho 4 \\
\hline Taxa_S & 18 & 31 & 9 & 8 \\
Individuals & 175 & 250 & 14 & 32 \\
Dominance_D & 0.2686 & 0.152 & 0.1429 & 0.2285 \\
Shannon_H & 1.943 & 2.476 & 2.069 & 1.683 \\
Margalef & 3.292 & 5.433 & 3.031 & 2.02 \\
Equitability_J & 0.6724 & 0.7211 & 0.9417 & 0.8092 \\
\hline
\end{tabular}




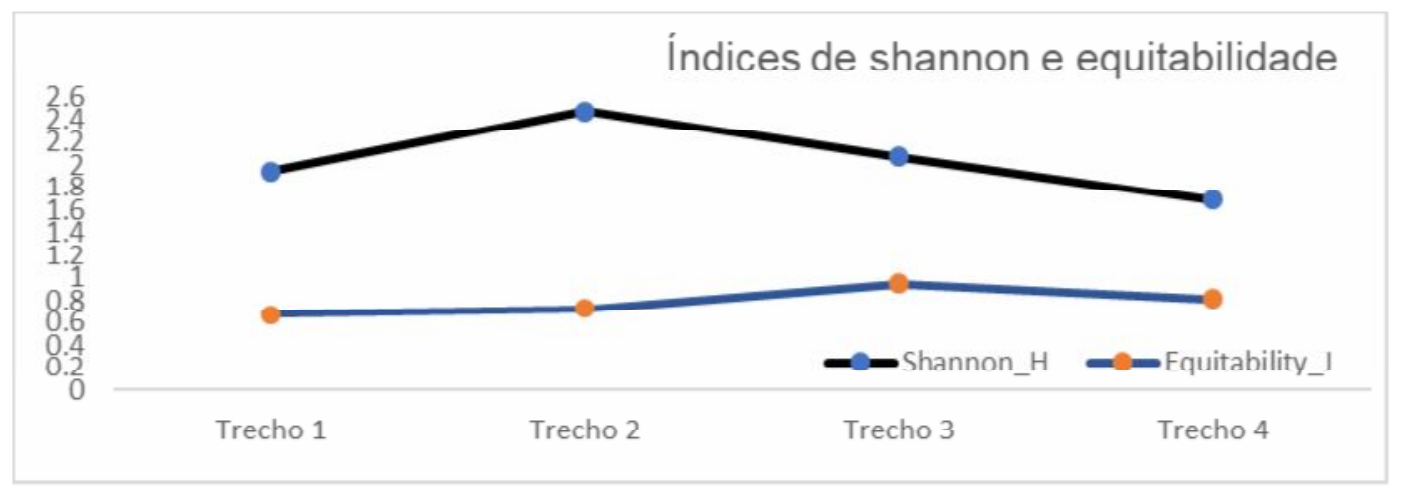

FIGURA 2. Índices de Shannon e Equitabilidade dos quatros trechos de coleta do Córrego Severo município de Alta Floresta - MT.

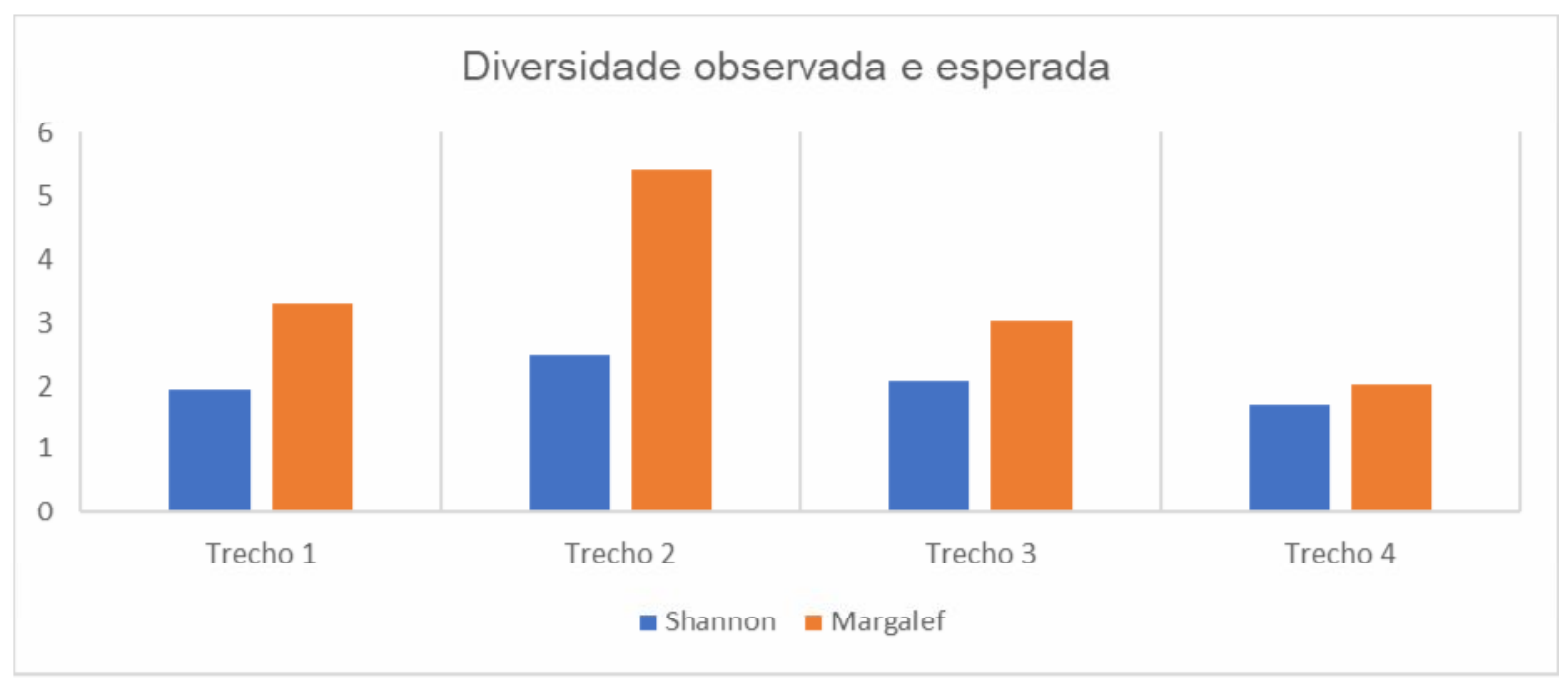

FIGURA 3. Índices de diversidade observada (Shannon) e diversidade esperada (Margalef), dos trechos de coleta do Córrego Severo.

$\mathrm{Na}$ análise de agrupamento foi utilizada a comparação por média (UPGMA), onde demostrou que os trechos 2 e 4 apresentaram maior similaridade, com correlação do coeficiente cofenetico de 0,9768 (Figura 4).

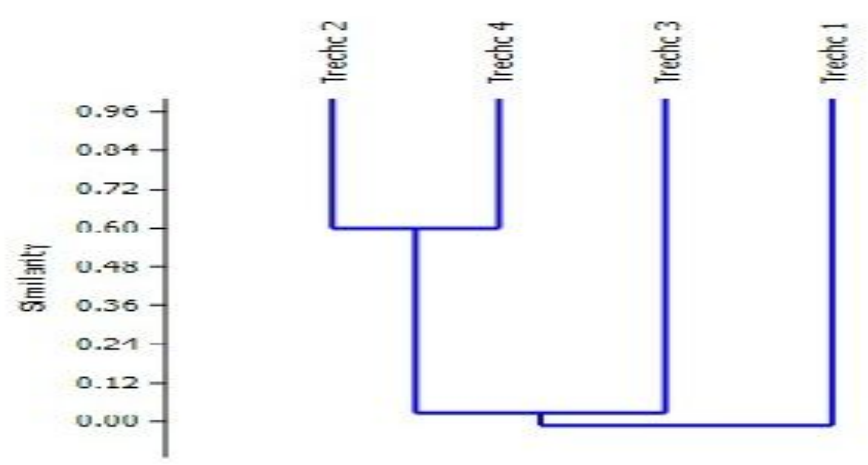

FIGURA 4. Analise de agrupamento de Cluster pelo método UPGMA dos pontos de coleta do Córrego Severo. 
A dominância da ordem Characiformes observada na coleta realizada Córrego Severo é um padrão comum dos córregos da região neotropical (CASATTI et al., 2001; BUCKUP et al., 2007). A prevalência desta ordem está relacionada com sua alta mobilidade no ambiente, em bueiros de riachos neotropicais observou o predomínio da ordem Characiformes e Siluriformes (MARIANO, 2012).

Nas coletas realizadas no Córrego Severo, ocorreu uma superioridade de espécies de pequeno porte, esse domínio ecológico ocorre devido sua fácil mobilidade em ambientes menores, também pode ser explicado por possuírem hábitos alimentares do tipo onívoro, o que facilita a captação dos recursos alimentares (CASTRO et al., 2004 e CASATTI et al., 2012).

Modificações antrópicas nos ecossistemas aquáticos podem causar impactos na diversidade de espécies modificando a estrutura da comunidade de peixes nos riachos e nos diversos ambientes (VILLÉGER et al., 2010; TERESA; CASATTI, 2012). Riachos com mata ripária degradada favorecem a ocorrência em alta frequência e abundância de espécies, que possuem grande plasticidade fenotípica e que são tolerantes às interferências antrópicas, como Knodus moenkhausii e Poecilia reticulata (CENEVIVA-BASTOS ; CASATTI, 2007 ; TERESA ; CARVALHO, 2008).

A espécie Poecilia reticulata, foi registrada no Córrego Severo o que indica a degradação do ambiente. A representatividade desta espécie é um forte indicativo de redução da integridade biológica do ecossistema (ALLAN ; FLECKER, 1993; FERREIRA ; CASATTI, 2006, CASATTI et al., 2009). Estas alterações antrópicas tornam a estrutura física do habitat um fator determinante na organização da comunidade aquática, podendo afetar a riqueza, abundância e composição das espécies (ROMERO et al., 2013; ROSENVALD, 2014).

\section{CONCLUSÃO}

A diversidade de espécies e a abundância de espécies de pequeno porte está relacionada com a antropização do córrego Severo devido à pouca cobertura vegetal. Para a manutenção da biodiversidade aquática são necessários a preservação de remanescentes florestais e os cursos d'agua que estes abrigam desta forma condicionando uma melhora no ecossistema aquático e preservando a biodiversidade dos peixes.

\section{AGRADECIMENTO}

Agradecemos a Dra. Solange Aparecida Arrolho, Coordenadora do Laboratório de Ictiologia da Amazônia Meridional pela colaboração.

\section{REFERÊNCIAS}

ALLAN, J.;FLECKER, A. S. Biodiversity conservation in running waters. BioScience, v. 43, $\quad$ n. $1, \quad$ p. 1993. http://www.eeb.cornell.edu/flecker/pdf/Allan\%20\&\%20Flecker\%201993_Biosci.pdf.

BARRELA, W.; PETRERE-JR., M.; SMITH, W. S.; MONTAG, L. F. As relações entre as matas ciliares, os rios e os peixes. In: RODRIGUES, R. R.; LEITÃO FILHO, H. F. (Ed.). Matas ciliares: conservação e recuperação. São Paulo: Edusp; Fapesp, p. 187-207. 2001. https://www.researchgate.net/publication/316101395_As_relacoes_entre_as_matas_ 
ciliares_os_rios_e_os_peixes.

BOJSEN, B. H.; BARRIGA, R. Effects of deforestation on fish community structure in Ecuadorian Amazon streams. Freshwater Biology, v. 47, n. 11, p. 2246-2260, 2002. DOI: 10.1046 / j.1365-2427.2002.00956.x.

BUCKUP, P. A.; MENEZES, N. A.; GHAZZI, M. S. Catálogo das espécies de peixes de água doce do Brasil. Rio de Janeiro: Museu Nacional, 2007. Disponível em:

https://www.researchgate.net/profile/Paulo_Buckup/publication/277475495_Buckup et_al_2007Catalogo_das_Especies_de_Peixes_de_Agua_Doce_do_Brasil//inks $/ 556$ b41c808aeab77722144ec/Buckup-et-al-2007-Catalogo-das-Especies-de-Peixes-deAgua-Doce-do-Brasil.pdf.

CASATTI, L., ROMERO, R. D. M., TERESA, F. B., SABINO, J., \& LANGEANI, F. Fish community structure along a conservation gradient in Bodoquena Plateau streams, central West of Brazil. Acta Limnologica Brasiliensia, 50-59. (2010). doi: 10.4322/actalb.02201007.

CASATTI, L.; TERESA, F.B.; GONÇALVES-SOUZA, T.; BESSA, E.; MANZOTTI, A.R. et al. From forests to cattail: how does the riparian zone influence stream fish? Neotropical Ichthyology, v. 10, n. 1, p. 205-214, 2012. http://dx.doi.org/10.1590/S1679-62252012000100020.

CASATTI, L.; FERREIRA, C. P.; LANGEANI, F.. A fish-based biotic integrity index for assessment of lowland streams in southeastern Brazil. Hydrobiologia, v. 623, n. 1, p. 173-189, 2009. DOI 10.1007/s10750-008-9656-x.

CASATTI, L; LANGEANI, F.; CASTRO, R. Peixes de riacho do Parque Estadual Morro do Diabo, bacia do alto rio Paraná, SP. Biota Neotropica, p. 1-15, $2001 .$. https://repositorio.unesp.br/handle/11449/22496

CASTRO, R., CASATTI, L., SANTOS, H. F., MELO, A. L., MARTINS, L. S., FERREIRA, K. M., ... \& ABREU, T. X. Estrutura e composição da ictiofauna de riachos da bacia do rio Grande no estado de São Paulo, sudeste do Brasil. Biota Neotropica, 4(1), 01-39. (2004). http://www.scielo.br/pdf/bn/v4n1/v4n1a06.pdf.

CENEVIVA-BASTOS, M; CASATTI, L. Oportunismo alimentar de Knodus moenkhausii (Teleostei, Characidae): uma espécie abundante em riachos do noroeste do Estado de São Paulo, Brasil. Iheringia: Série Zoologia, p. 7-15, 2007. https://repositorio.unesp.br/handle/11449/22505.

FERREIRA, C. P.; CASATTI. Influência da estrutura do habitat sobre a ictiofauna de um riacho em uma micro- bacia de pastagem, São Paulo Brasil. Revista Brasileira de Zoologia, v.23, n.3, p. 2006. http://www.scielo.br/pdf/rbzool/v23n3/a06v23n3.

GÉRY, J. Characoids of the world. Neptune City, TFH Publications. 1977. 
KARR, J.R.; DUDLEY, D.R. Ecological perspective on water quality goals. Environmental management, v. 5, n. 1, p. 55-68, 1981. https://link.springer.com/article/10.1007\%2FBF01866609? LI=true.

LORION, C. M.; KENNEDY, B. P. Riparian forest buffers mitigate the effects of deforestation on fish assemblages in tropical headwater streams. Journal of Applied Ecology, v. 19, n. 2, p. 468-479, 2009. DOI: 10.1890/08-0050.1

MACHADO, ANGELO BARBOSA MONTEIRO; DRUMMOND, GLÁUCIA MOREIRA; PAGLIA, ADRIANO PEREIRA. Livro vermelho da fauna brasileira ameaçada de extinção. In: Livro vermelho da fauna brasileira ameaçada de extinção. MMA; Fundação Biodiversitas, p12-16.2008. http://www.mma.gov.br/estruturas/sbf2008_dcbio/_publicacao/147_publicacao31032 009031646.pdf.

MARIANO, J.R.; MAKRAKIS, M.C.; KASHIWAQUI, E.A.L.; CELESTINO, E.F.; MAKRAKIS, S.; Longitudinal habitat disruption in Neotropical streams: fish assemblages under the influence of culverts. Neotropical Ichthyology, v. 10, n. 4, p. 771-784, 2012. http://dx.doi.org/10.1590/S1679-62252012000400010.

MIRANDE, J. M. Phylogeny of the family Characidae (Teleostei: Characiformes): from characters to taxonomy. Neotropical Ichthyology, v.8, p.385-568. 2010. http://dx.doi.org/10.1590/S1679-62252010000300001.

REIS, R. E. Conserving the freshwater fishes of South America. International Zoo Yearbook 47, 1-6. (2013). DOI:10.1111/izy.12000.

REIS, R. E.; KULLANDER, S. O.; FERRARIS, C. J. Check list of the freshwater fishes of South and Central America. Edipucrs, 2003. https://s3.amazonaws.com/academia.edu.documents/41823863/Genera_incertae_se dis in Characidae20160131-31041-

7bw4td.pdf?AWSAccessKeyld=AKIAIWOWYYGZ2Y53UL3A\&Expires $=1504907999 \&$ Signature $=$ LrSgM6\%2BVUpRaYL7k6\%2FWsk7\%2FWvn0\%3D\&response-contentdisposition=inline\%3B\%20filename\%3DGenera incertae_sedis_in_Characidae.pdf

ROMERO, R.M.; CENEVIVA-BASTOS, M.; BAVIERA, G.H.; CASSATI, L. Community structure of aquatic insects (Ephemeroptera, Plecoptera, and Trichoptera) in Cerrado streams of Paraguay, Paraná, and São Francisco river basins. Biota Neotropica, v. 13, n. 1, p. 97-107, 2013. http://dx.doi.org/10.1590/S1676-06032013000100011.

ROSENVALD, R.; JÄRVEKÜLG, R.; LÕHMUS, A. Fish assemblages in forest drainage ditches: Degraded small streams or novel habitats?. LimnologicaEcology and Management of Inland Waters, v. 46, p. 37-44, 2014. https://doi.org/10.1016/j.limno.2013.12.004.

TERESA, F. B.; CASATTI, L. Influence of forest cover and mesohabitat types on functional and taxonomic diversity of fish communities in Neotropical lowland streams. Ecology of Freshwater Fish, v. 21, n. 3, p. 433-442, 2012. DOI: 10.1111 / 
j.1600-0633.2012.00562.x.

TERESA, F. B.; CARVALHO, F.R. Feeding association between benthic and nektonic neotropical stream fishes. Neotropical Ichthyology, v. 6, n. 1, p. 109-111, 2008. http://dx.doi.org/10.1590/S1679-62252008000100013.

TERESA, F. B.; CASATTI. Importância da vegetação ripária em região intensamente desmatada no sudeste do Brasil: um estudo com peixes de riacho. Pan-American Journal of Aquatic Sciences, p. $444 \quad-4453,2010$. https://repositorio.unesp.br/bitstream/handle/11449/22556/ZOORECZOOR14711079 221. pdf? sequence $=1$ \&isAllowed $=y$.

TOWNSEND, C. R.; BEGON, M.; HARPER, J.L. Fundamentos em ecologia.3. Ed. Porto Alegre, 576 p, 2010.

VILLÉGER, S., MIRANDA, J. R., HERNÁNDEZ, D. F., \& MOUILLOT, D. Contrasting changes in taxonomic vs. functional diversity of tropical fish communities after habitat degradation. Ecological Applications, 20(6), 1512-1522. (2010). http://villeger.sebastien.free.fr/pdf\%20publis/Villeger\%20et\%20al.\%20(2010)\%20Ecol \%20Appl\%20.pdf. 